\section{(A) Check for updates}

Cite this: Nanoscale, 2021, 13, 6266

\title{
Correction: Tuning trion binding energy and oscillator strength in a laterally finite 2D system: CdSe nanoplatelets as a model system for trion properties
}

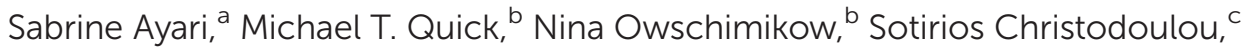 \\ Guillaume H. V. Bertrand, ${ }^{\mathrm{d}}$ Mikhail Artemyev, ${ }^{\mathrm{e}}$ Iwan Moreels, ${ }^{f}$ Ulrike Woggon, ${ }^{\mathrm{b}}$ \\ Sihem Jaziria, and Alexander W. Achtstein ${ }^{\mathrm{a}}$
}

DOI: $10.1039 / \mathrm{d} 1 \mathrm{nr} 90043 a$

Correction for 'Tuning trion binding energy and oscillator strength in a laterally finite 2D system: CdSe nanoplatelets as a model system for trion properties' by Sabrine Ayari et al., Nanoscale, 2020, 12.

rsc.li/nanoscale 14448-14458, DOI: 10.1039/D0NR03170D

The authors regret that typographic errors were present in some of the un-numbered equations in the Methods section on page 14454 of the original manuscript. The corrected paragraph should read as below. The errors do not affect the conclusions or results and discussion in the manuscript, as the modelling used the correct equations; only the display of the equations is changed.

'Actually, the electric force lines emerging from charges within a semiconductor nanoparticle pass through the surrounding medium, having a smaller dielectric constant than the semiconductor. Therefore, in order to take the dielectric screening of Coulomb interaction properly into account, the electron-hole direct Coulomb interaction is treated here using a Rytova-Keldysh potential $\hat{V}_{\mathrm{c}}\left(\boldsymbol{\rho}_{\mathrm{e}}-\boldsymbol{\rho}_{\mathrm{h}}\right)=-\frac{e^{2}}{2 \pi \varepsilon_{\text {env }}} \int \frac{e^{i q\left(\rho_{\mathrm{e}}-\rho_{\mathrm{h}}\right)} d^{2} q}{q\left(1+q r_{\mathrm{s}}\right)}$ according to the widely accepted approach (Ref. 7,31,32,51,74-77 in the original manuscript, published here as Ref. 1-8, respectively). However to avoid the divergence of the integral of the exponential function in the Keldysh potential, we can construct an approximate expression for $\hat{V}_{\mathrm{c}}(\rho)$ in terms of elementary functions

$$
\hat{V}_{\mathrm{c}}\left(\boldsymbol{\rho}_{\mathrm{e}}-\boldsymbol{\rho}_{\mathrm{h}}\right)=\frac{e^{2}}{2 \varepsilon_{\mathrm{env}} r_{\mathrm{s}}}\left[\ln \left(\frac{\left|\boldsymbol{\rho}_{\mathrm{e}}-\boldsymbol{\rho}_{\mathrm{h}}\right|}{\left|\boldsymbol{\rho}_{\mathrm{e}}-\boldsymbol{\rho}_{\mathrm{h}}\right|+r_{\mathrm{s}}}\right)+(\gamma-\ln (2)) e^{\frac{-\left|\boldsymbol{\rho}_{\mathrm{e}}-\rho_{\mathrm{h}}\right|}{r_{\mathrm{s}}}}\right]
$$

(See ref. 8 below for details). This potential is better than using an unscreened vacuum Coulomb potential together with envelope functions in $z$-direction, the standard approach for quantum wells. Here, $r_{\mathrm{s}}=\varepsilon_{\mathrm{cdse}} Z_{0} /\left(2 \varepsilon_{\text {env }}\right)$ is the dielectric screening length, $Z_{0}=\left(a_{0} / 2\right) \times 4.5 \mathrm{ML}$ is the platelet thickness and $\gamma$ is the Euler constant'.

The Royal Society of Chemistry apologises for these errors and any consequent inconvenience to authors and readers.

\section{Notes and references}

7 S. Ayari, A. Smiri, A. Hichri, S. Jaziri and T. Amand, Phys. Rev. B, 2018, 98, 205430.

31 A. Hichri, I. B. Amara, S. Ayari and S. Jaziri, J. Phys.: Condens. Matter, 2017, 29, 435305.

32 M. Richter, Phys. Rev. Mater., 2017, 1, 016001.

\footnotetext{
${ }^{a}$ Laboratoire de Physique des Materiaux, Faculte des Sciences de Bizerte, Universite de Carthage, Jarzouna 7021, Tunisia

${ }^{b}$ Institute of Optics and Atomic Physics, Technische Universität Berlin, Strasse des 17. Juni 135, 10623 Berlin, Germany. E-mail: achtstein@tu-berlin.de ${ }^{c}$ ICFO-Institut de Ciencies Fotoniques, 08860 Castelldefels, Barcelona, Spain

${ }^{d}$ CEA Saclay, 91191 Gif-sur-Yvette, France

${ }^{e}$ Research Institute for Physical Chemical Problems of Belarusian State University, 220006 Minsk, Belarus

${ }^{f}$ Department of Chemistry, Ghent University, Krijgslaan 281 - S3, 9000 Gent, Belgium

${ }^{g}$ Laboratoire de Physique de la Matiere Condensee, Departement de Physique, Faculte des Sciences de Tunis, Campus Universitaire1060 Tunis, Tunisia
} 
51 J. F. Specht, R. Scott, M. C. Castro, S. Christodoulou, G. H. Bertrand, A. Prudnikau, A. Antanovich, L. Siebbeles, N. Owschimikow, I. Moreels, et al., Nanoscale, 2019, 11, 12230-12241.

74 A. Hichri, I. Ben Amara, S. Ayari and S. Jaziri, J. Appl. Phys., 2017, 121, 235702.

75 N. S. Rytova, 2018, arXiv preprint arXiv:1806.00976.

76 A. Chernikov, T. C. Berkelbach, H. M. Hill, A. Rigosi, Y. Li, O. B. Aslan, D. R. Reichman, M. S. Hybertsen and T. F. Heinz, Phys. Rev. Lett., 2014, 113, 076802.

77 P. Cudazzo, I. V. Tokatly and A. Rubio, Phys. Rev. B: Condens. Matter Mater. Phys., 2011, 84, 085406. 